\title{
Ultrasonic analysis of tungsten monoblock divertor mock-ups after high heat flux test
}

\author{
Giacomo Dose ${ }^{\mathrm{a}}$, Selanna Roccella ${ }^{\mathrm{b}}$, Marianne Richou ${ }^{\mathrm{c}}$, Franklin Gallay $^{\mathrm{c}}$, Eliseo Visca ${ }^{\mathrm{b}}$, \\ Henri Greuner $^{d}$, Francesco Romanelli ${ }^{a}$, Katja Hunger ${ }^{d}$, Tom Barrett ${ }^{e}$ and Jeong-Ha You ${ }^{d}$ \\ a Università di Roma “Tor Vergata”, Dipartimento di Ingegneria Industriale, Via del Politecnico 1, 00133 Rome, Italy \\ ${ }^{b}$ ENEA, Department of Fusion and Technology for Nuclear Safety and Security, Frascati, Italy \\ ${ }^{c}$ CEA, IRFM, F-13108 Saint-Paul-Lez-Durance, France \\ ${ }^{d}$ Max-Planck-Institut für Plasmaphysik, Boltzmannstr. 2, 85748 Garching, Germany \\ ${ }^{e}$ CCFE, Culham Science Centre, Abingdon, Oxon, OX14 3DB, United Kingdom
}

\begin{abstract}
In this paper, a comparison is presented among the ultrasonic measures of different $\mathrm{W}$-monoblock mock-ups following high heat flux tests. The DEMO reference solution for the divertor targets (ITER-like) is compared with two other concepts, in which the interlayer between monoblocks and tube has been engineered to increase the performance of the component (Thermal Break Interlayer, Thin Graded Interlayer). Ultrasonic measurements have been performed at the depths of interest. The reflected signal amplitude and time of flight at defined depths are used to determine the shape and entity of the defect, when present. For each concept, the most frequently observed defects due to thermal loading are then identified. Preliminary post-mortem analysis confirms the results from the ultrasonic tests, proving the defect imaging capability of such non-destructive technique for advanced W-monoblock concepts.
\end{abstract}

Keywords: ultrasonic testing, non-destructive analysis, high heat flux, functionally graded interlayer, thermal break.

\section{Introduction}

In a fusion reactor, heat exhaust is one of the most challenging engineering issues, also due to the high heat flux (HHF) expected on the divertor targets. For DEMO, loads from $10 \mathrm{MWm}^{-2}$, for quasi-stationary operation, to $17-21 \mathrm{MWm}^{-2}$, when slow transient events occur, are predicted locally in the narrow region near the strike point [1]. Among the different layouts, the tungsten (W) monoblock design represents one of the most suitable technological solutions for plasma facing components, since it has already met the present ITER design requirements [2]. However, further research is required to investigate improved solutions able both to operate at DEMO conditions and to increase the expected lifetime of the component. In this context, new water-cooled divertor concepts are being developed as part of the research activity carried out by the EUROfusion Consortium. Therefore, several W monoblock mock-ups with innovative design have been manufactured by the involved partners, and subsequently tested with DEMO relevant cyclic heat loading in the HHF test facility GLADIS at IPP Garching [3]. Among others, two concepts, namely Thermal Break Interlayer (TBI) and Thin Graded Interlayer (TGI), have been developed and compared to the baseline reference of water-cooled divertor targets (ITER-like, IL). In the TBI concept [4], being developed at CCFE, the copper interlayer presents 6 radial spokes at the HHF side, as well as an additional groove on the low flux side at the axis of symmetry. In this way, the interlayer acts as a thermal barrier that helps the repartition, around the circumference of the pipe, of the heat flux that would otherwise intensify on the plasma-facing side of the heat sink. On the plasma side, the $\mathrm{W}$ block is divided in two halves in order to decrease the stress inside the armor during cycled loading. The TGI is a concept developed at CEA Cadarache, where a solid $\mathrm{W}$ block is bonded to the $\mathrm{CuCrZr}$ tube using a graded interlayer where the concentration varies in $25 \mu \mathrm{m}$ from $0 \% \mathrm{~W}(100 \% \mathrm{Cu})$ to $100 \% \mathrm{~W}(0 \% \mathrm{Cu})$ [5]. The aim of the concept is to reduce both the amount of copper used, since it is a material prone to embrittlement due to fast neutron, and the thermal resistance of the component, by minimizing the heat conduction path. After the HHF test campaign it is crucial to assess the integrity of each mockup and to identify the critical features of each target concept. For this purpose, ultrasonic test (UT) was performed at ENEA Frascati on all the mock-ups (6 IL, 4 TGI, 6 TBI), both before and after the HHF tests, in order to observe the formation of new defects as well as the evolution of pre-existing ones. UT was demonstrated to be able to detect defects and their location inside IL components with good accuracy [6]. However, the UT capability of detecting defects for innovative concepts is still to be confirmed. Compared to other non-destructive techniques, UT is particularly appropriate to test W monoblock mock-ups due to the opacity of $\mathrm{W}$ to X-rays, as well as the density and homogeneity of both $\mathrm{W}$ and $\mathrm{CuCrZr}$, which result in clear transmitted pressure signals. The UT system used allows a direct imaging of the defects inside the component with a resolution of $500 \mu \mathrm{m}$. In this paper, we present a comparison between ultrasonic scans, performed after the HHF test campaign, of three representative mock-ups of the studied concepts: IL, TBI and TGI. A comparison with preliminary metallographic images is performed in order to validate UT conclusions.

\section{Materials and methods}

\subsection{Description of the mock-ups}

Block dimensions as well as inner and outer diameter of the heat sink pipe for all concepts are reported in fig. 1 . 
All monoblocks have $4 \mathrm{~mm}$ of thickness along the z-axis for all layouts. A swirled tape is also fixed inside the pipe for all samples. The IL mock-up, manufactured by ENEA Frascati, consists of 12 blocks which are joined to the $\mathrm{CuCrZr}$ tube by means of Hot Radial Pressing (HRP; 600 ${ }^{\circ} \mathrm{C}, 50 \mathrm{MPa}, 2 \mathrm{~h}$ ) [7]. This concept provides a pure $\mathrm{Cu}$ interlayer of $0.5 \mathrm{~mm}$ thickness, which has been casted on the block. The mock-up with TGI is made of 10 blocks and is realized by CEA Cadarache. The interlayer has a thickness of $25 \mu \mathrm{m}$ and is obtained through PVD. The deposit was performed inside the bore hole of the $\mathrm{W}$ monoblock, which is then joined to the $\mathrm{CuCrZr}$ pipe through Hot Isostatic Pressing (HIP; $950{ }^{\circ} \mathrm{C}, 120 \mathrm{MPa}, 2$ h). Thermal aging has been applied on the mock-up (475 ${ }^{\circ} \mathrm{C}, 3 \mathrm{~h}$ ). The component with the TBI has 10 blocks and is fabricated by CCFE using a two-stage vacuum braze process. First, a pure copper sleeve is brazed on the $\mathrm{CuCrZr}$ tube, providing the interlayer material. Then the copper is machined, obtaining the structured interlayer geometry. The $\mathrm{W}$ blocks, provided with a thin casted $\mathrm{Cu}$ layer into the central bore, are than bonded to the first assemble through a second $\mathrm{Cu}$-to- $\mathrm{Cu}$ brazing process $\left(1000{ }^{\circ} \mathrm{C}\right)$. A precipitation hardening cycle is applied $(475$ ${ }^{\circ} \mathrm{C}, 2 \mathrm{~h}$ ). The overall TBI thickness is $2 \mathrm{~mm}$.

\subsection{HHF testing}

The HHF tests were performed at the neutral beam facility GLADIS at IPP Garching. The first step of the test procedure consists in an initial screening from 6 to 20 (or 25) $\mathrm{MWm}^{-2}$ and 100 cycles at $10 \mathrm{MWm}^{-2}$. The coolant inlet condition is $20^{\circ} \mathrm{C}, 10$ bar and $12 \mathrm{~m} / \mathrm{s}$. The second step of the HHF tests is conducted at DEMO relevant water conditions: $130{ }^{\circ} \mathrm{C}, 40 \mathrm{bar}, 16 \mathrm{~m} / \mathrm{s}$. This section starts with an additional screening up to $20 \mathrm{MWm}^{-2}$, and ends with the thermal fatigue test up to 500 cycles at 20 $M W m^{-2}$. Each beam pulse lasts $10 \mathrm{~s}$, time interval in which the loaded sample reaches the thermal equilibrium.

\subsection{Ultrasonic test}

UT was performed at the laboratories of ENEA Frascati. The method used was a pulse-echo water gap technique. The test is conducted by inserting an ultrasonic pulser/receiver probe inside the $\mathrm{CuCrZr}$ pipe with water fill. A handling system with two degrees of freedom, one translation and one rotation, operates the probe along the whole sample. The piezoelectric crystal inside the probes has a diameter of about $3 \mathrm{~mm}$ and works at frequencies of about $15 \mathrm{MHz}$. The probes are cylindrically shaped with the crystal placed laterally, normal to the radial direction of the component. In order to identify the defects due to the HHF tests, the ultrasonic scans are compared to the ones performed on the same samples before the thermal
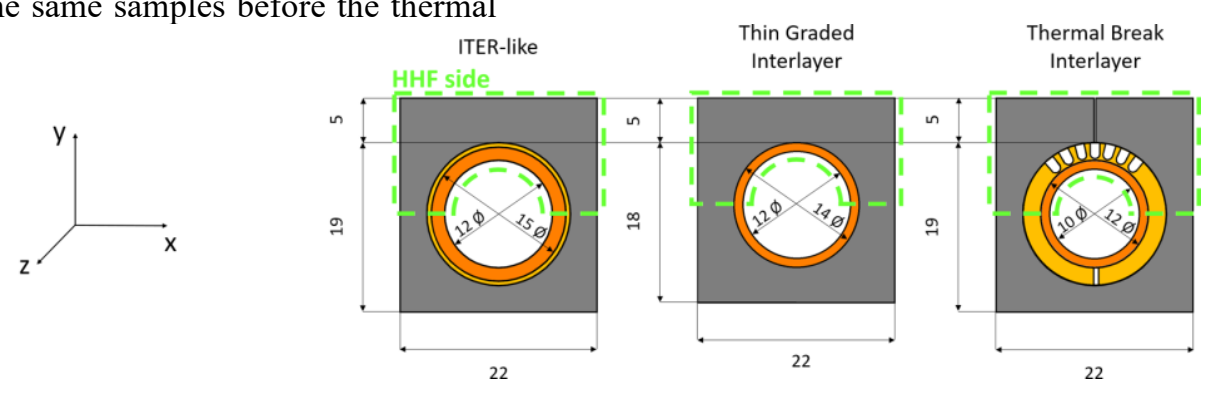
2. The maps are reported for a $\triangle T O F$ around the bonding interface between $\mathrm{CuCrZr}$ pipe and the $\mathrm{Cu}$ interlayer (HRP interface), since no defects were detected at other interfaces. The outer surface of the naked tube appears as red lateral bands in the $\mathrm{C}$-scan, and it is detected, as expected, at $T O F=1.50 \mathrm{~mm}$ (red colour in D-scan). In the D-scan, we observe also reds spots inside the sample, which correspond to defects at the HRP interface. These indications are manufacturing defects and therefore were present before the HHF tests. Their dimension did not increase after the thermal fatigue campaign. From the Cscan, we detect small debonding at the sides of all monoblocks that appeared at the HHF region. Their maximum extension along the pipe axis is of $1.3 \mathrm{~mm}$ on average, shared between blocks. This indication is detected only after the thermal cycles and it is located at the HRP interface. In the C-scan, we observe also the appearance of small pores in the HHF region of the sample. A better view of this indication is available in the D-scan where such porosity appears as white spots, which means that the pores are located inside the $\mathrm{Cu}$ interlayer ( $\sim 0.3 \mathrm{~mm}$ from HRP interface). Preliminary post-mortem analysis confirms UT result (Fig. 5), where debonding at ends of blocks and porosity are observed.

No defects are detected in the mock-up with TGI both inside $\mathrm{W}$ and at the HIP interface, which did not show any debonding or failure of the graded material as reported in figure 3(right). The TGI in fact did not affect the detection

Fig. 1. Block dimensions and inner/outer diameter of $\mathrm{CuCrZr}$ tube for each studied concept (interlayer represented in yellow). 


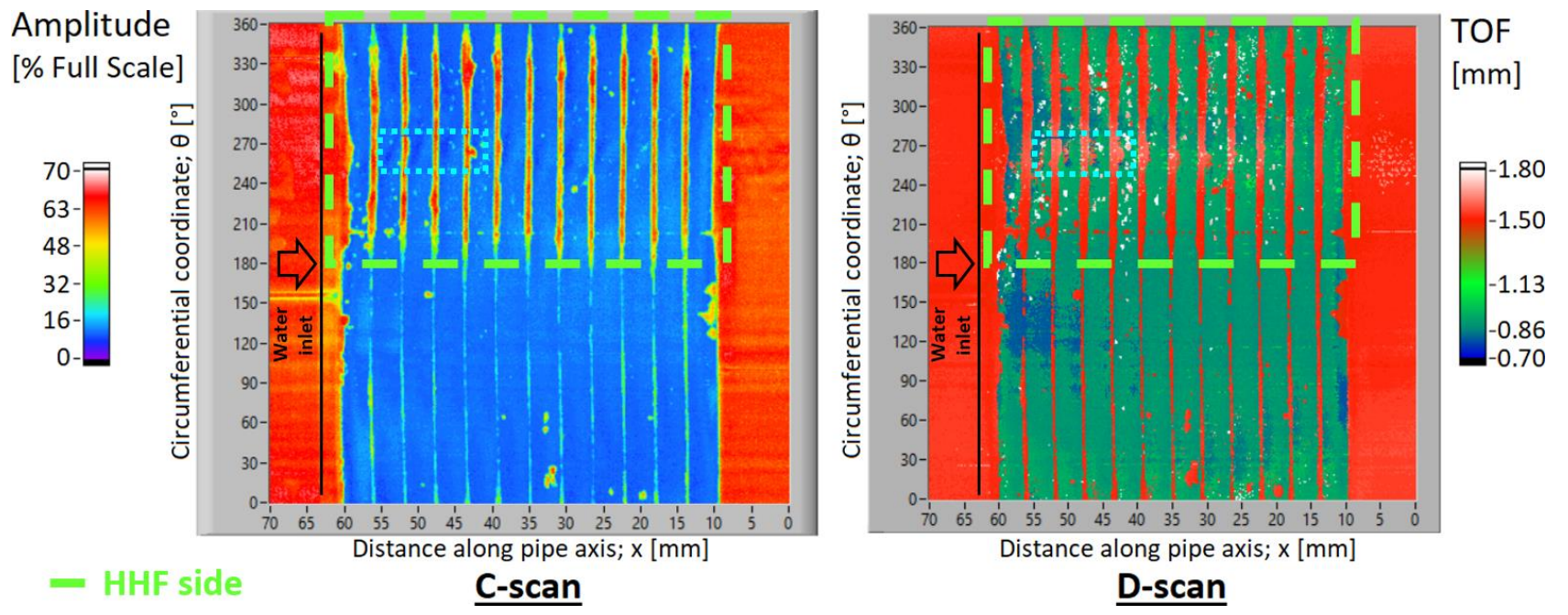

Fig. 2. Scans of the IL mock-up after HHFT. $\triangle T O F=0.80-1.83 \mathrm{~mm}(\mathrm{v}=5000 \mathrm{~m} / \mathrm{s}) ;$ around $\mathrm{CuCrZr} /$ interlayer interface. $\mathrm{C}$-scan: blue colour corresponds to good bonding; green to red is for an indication of a defect (debonding at sides of blocks in HHF region, spots). D-scan: In red are all signals measured at the depth of the $\mathrm{CuCrZr}$ /interlayer interface (at $1.5 \mathrm{~mm}$ ); white spots represent porosity inside the interlayer (at $\sim 1.8 \mathrm{~mm}$ ) which is almost entirely localized in the HHF region. In light blue dashed rectangle are the defects also showed in Fig. 5.
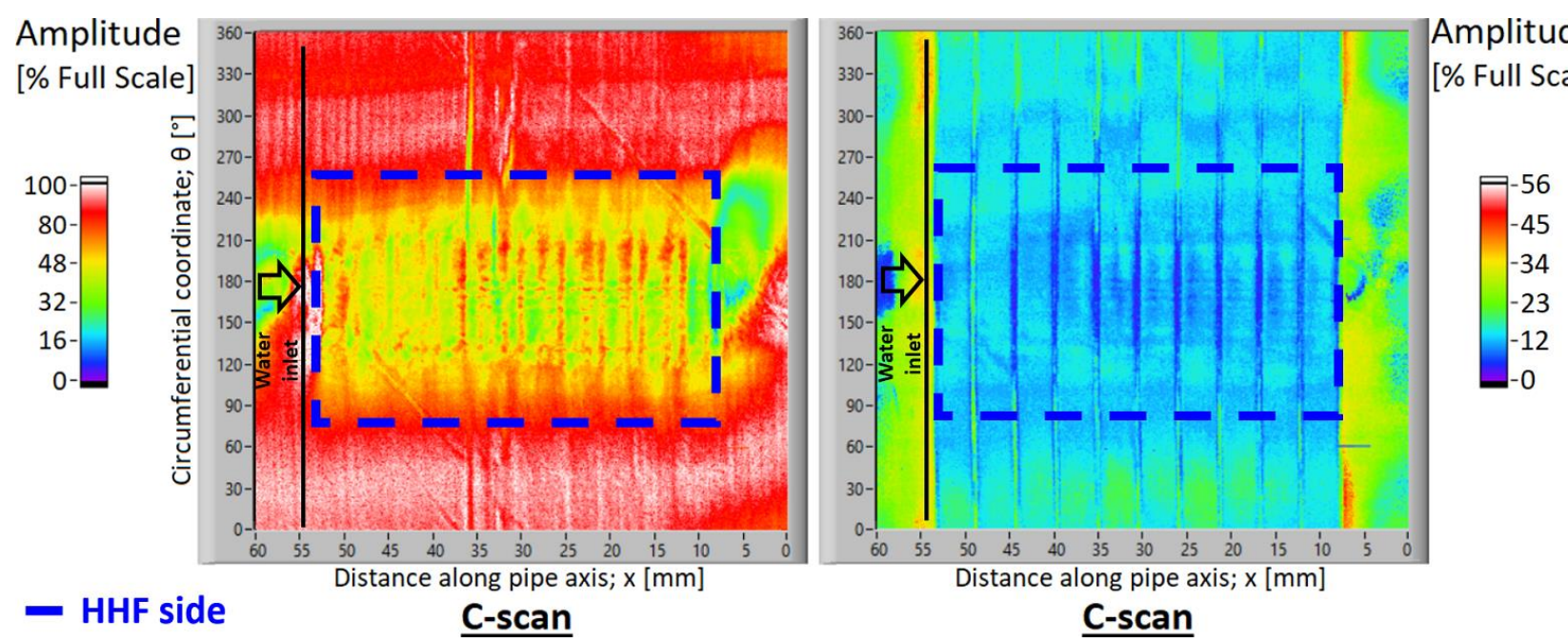

Fig. 3. C-scans of the TGI mock-up after HHFT. Left: $\triangle T O F=0.00-0.40 \mathrm{~mm}(\mathrm{v}=5000 \mathrm{~m} / \mathrm{s})$; water/CuCrZr interface. Modification of pipe inner surface in HHF region. Right: $\triangle T O F=0.90-1.15 \mathrm{~mm}(\mathrm{v}=5000 \mathrm{~m} / \mathrm{s})$; HIP interface. No defects.
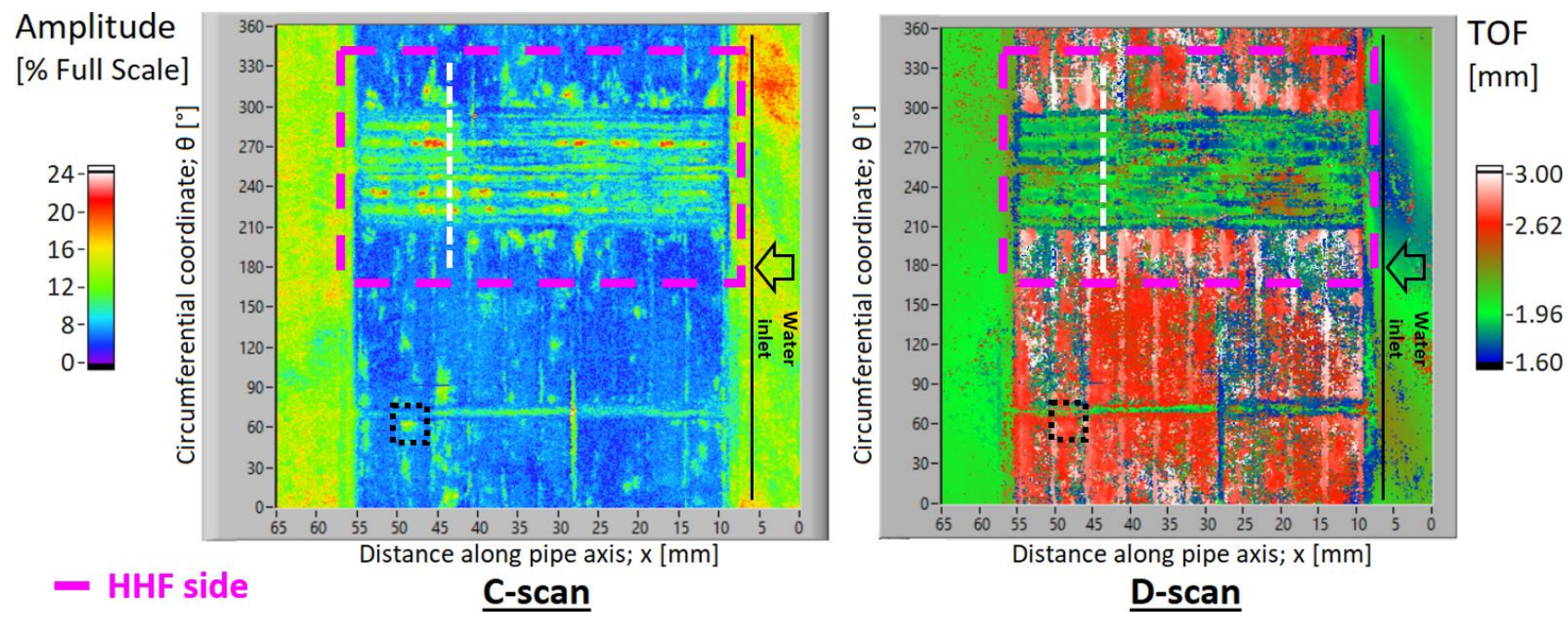

Fig. 4. Scans of the TBI mock-up after HHFT. $\triangle T O F=1.65-3.30 \mathrm{~mm}(\mathrm{v}=5000 \mathrm{~m} / \mathrm{s})$; interlayer/block interface. C-scan: blue colour represents good bonding; green/red is for indication of defects (failure of spokes, porosity, debonding at sides of spokes region); lateral green bands refer to naked pipe. D-scan: blue to green is for a depth inside interlayer $(1.60-1.96 \mathrm{~mm})$; red to white is for indications at the second $\mathrm{Cu}$-to-Cu brazing interface $(2.62-3.00 \mathrm{~mm})$. In black dashed rectangle is the defect at $2^{\text {nd }}$ brazing int. showed in Fig. 7 (left). The dashed white line $\mathrm{x}=43 \mathrm{~mm} ; \Delta \theta=175^{\circ}-335^{\circ}$ refers to the B-scans in Fig. 6. 


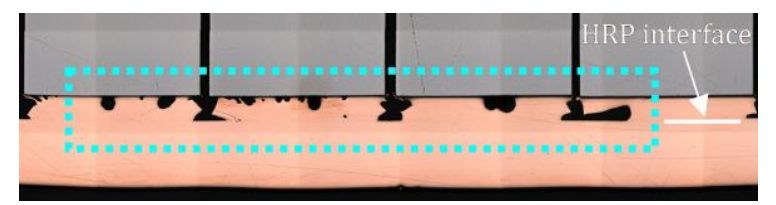

Fig. 5. Defects at blocks 2 to 5 at HHF region (see Fig.2)

of the HIP interface, probably due to its small thickness. In figure 3 (left) the $\mathrm{C}$-scan at the interface between water and $\mathrm{CuCrZr}$ shows the morphology of the inner surface of the pipe. We observe an apparent roughening of the surface at the HHF side of the component. From the sole ultrasound test, it is difficult to have a clear interpretation of this measurement. A possible speculation is that it could be a corrosion pattern of the $\mathrm{CuCrZr}$ due to the DEMO-relevant hydraulic condition. In the same region, we also observe a mild thickening of the tube of about $\sim 150 \mu \mathrm{m}$ (not shown in scans). This could be related to plastic deformation or to some defects inside the pipe that ultrasound cannot detect (e.g. microporosity). Further study is then required, as for example destructive metallographic analyses (ongoing). A similar indication, of both the pattern and the thickening of the tube plus interlayer thickness, is detected also in the IL mock-up, but not in the one with TBI.

The scans of the mock-up with TBI (Fig. 4) are reported at a $\triangle T O F$ that contains the interface between the $\mathrm{Cu}$ interlayer and the $\mathrm{W}$ block ( $2^{\text {nd }}$ brazing interface). In the $\mathrm{C}$-scan, we identify three main critical features. The first is the indications from the copper radial spokes (coloured green to red; C-scan). The morphology of these defects is difficult to determine due to the complex geometry of the sample. However, this indication was not present before HHF tests, as show in the B-scans in Fig. 6, and therefore we are confident that, in large part, the spokes were interrupted due to thermal fatigue cycling. Second type of defect that appeared after HHF tests, is a debonding between the $\mathrm{Cu}$ interlayer and $\mathrm{W}$ block, which is localized at the sides of the spokes region. In the $\mathrm{C}$-scan this appears as yellow/green spots at $185^{\circ}<\theta<200^{\circ} ; 290^{\circ}<\theta<$ $330^{\circ}$. This is the region of the component where the heat flux would intensify if no flux were exhausted through the spokes. The last defects consist of porosity at the second $\mathrm{Cu} / \mathrm{Cu}$ interface, represented as distributed green spots in the C-scan. Some pores where present before HHF tests, but the majority was generated during the thermal cycles. The indications inside the spokes appear in the D-scan at a depth from 1.60 to $1.96 \mathrm{~mm}$ (green/blue), while all other defects are detected between 2.62 and $3.00 \mathrm{~mm}$ (red/white; $2^{\text {nd }}$ brazing interface). Preliminary postmortem analysis confirms UT results by showing porosity at the second brazing interface (Fig. 7) and failure of the copper spokes. For the defects near the spoke region dedicated cuts are yet to be performed.

\section{Conclusions}

A comparison between three water-cooled Wmonoblock concepts has been performed. UT results for the different designs have been analysed before and after HHF tests. After thermal fatigue, the IL mock-up shows debonding at the sides of each block in the HHF region at the HRP interface. Manufacturing defects at the same

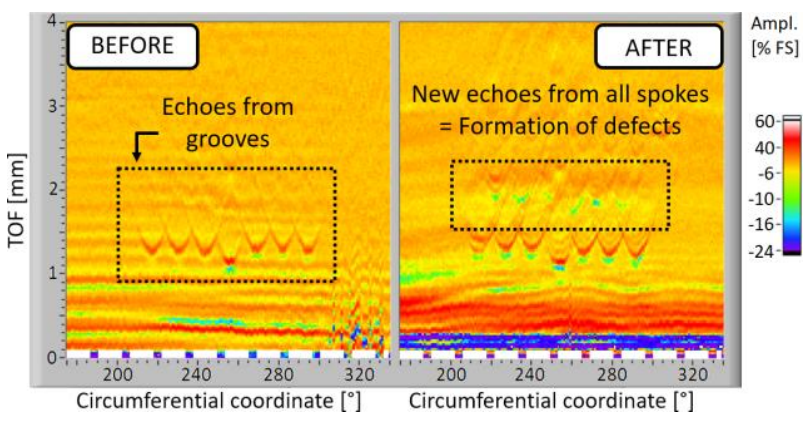

Fig. 6. B-scan before and after HHF tests along the dashed white line in Fig.4.

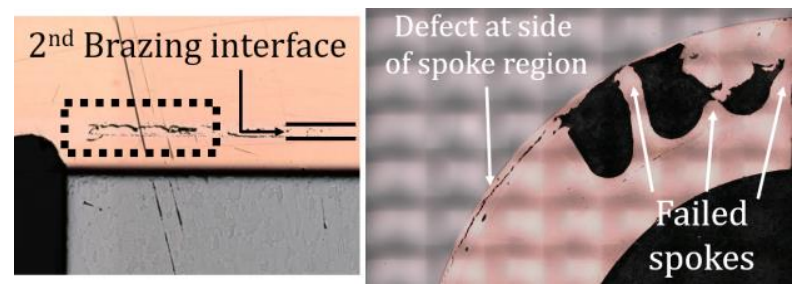

Fig. 7. (TBI) Left: Defect at block 9 at low flux side (see Fig. 4). Right: Defects in the right half of block 8.

interface did not increase their dimension after HHF tests. Porosity was detected in the casted $\mathrm{Cu}$ interlayer and increased in size at the HHF region after loading. The mock-up with the TGI showed no debonding at the HIP interface as well as no failure of the graded material. For both IL and TGI samples (and not for the TBI one), modification of the inner surface of the pipe was detected on the HHF side after thermal fatigue tests, as well as an increased thickness $(\sim 150 \mu \mathrm{m})$ of the pipe in the same region. In the mock-up with the TBI, failures of the copper spokes are detected after HHF tests. In addition, localized debonding at the second $\mathrm{Cu}$-to- $\mathrm{Cu}$ brazing interface appeared near the spokes region. Increased porosity is diffusely detected at the same interface as spread spots in the whole mock-up. Preliminary post-mortem analysis proves the UT capability in imaging defects with accuracy also for advanced W-monoblock divertor concepts.

\section{Acknowledgments}

This work has been carried out within the framework of the EUROfusion Consortium and has received funding from the Euratom research and training programme 20142018 under grant agreement No 633053. The views and opinions expressed herein do not necessarily reflect those of the European Commission.

\section{References}

[1] J.H. You et al., Nuclear Materials and Energy 9 (2016) 171176.

[2] Z. Sun et al., Fusion Engineering and Design 121 (2017) 6069.

[3] H. Greuner et al., Journal of Nuclear Materials 367-370 (2007) 1444-1448.

[4] M. Fursdon et al., Physica Scripta T170 (2017) 014042.

[5] M. Richou et al., Physica Scripta T170 (2017) 014022.

[6] S. Roccella et al., Fusion Engineering and Design 84 (2009) 1639-1644.

[7] E. Visca et al., Fusion Engineering and Design 75-79 (2005) 485-489. 